\title{
Solving the Schrödinger Equation Using Anharmonic Potentials and a Variational Quantum Monte Carlo Method
}

\author{
Maurício G. Rodrigues, Régis C. Leal \& Rogério Custodio
}

\section{Introduction}

Oscillators are important models in quantum systems because they are good approximation for different problems. The harmonic oscillator is the first approximation used for the vibrational spectroscopy models. ${ }^{1,2}$ In this model, Hooke's law defines the potential energy operator:

$$
\hat{V}(x)=\frac{1}{2} k x^{2}
$$

where $\mathrm{k}$ is the force constant and depends on the nature of the bonded atoms and $\mathrm{x}$ is the vibration coordinate.

A more realistic model to describe molecular vibrations is given by the anharmonic oscillator. In this case, many different forms of analytical operators have been proposed. One of the most popular is the well-known Morse potential, ${ }^{1}$

$$
\hat{V}(x)=D_{e}\left(1-e^{-\beta x}\right)^{2}
$$

where De is the molecular dissociation energy and $\beta$ is a parameter associated with the curvature of $\mathrm{V}(\mathrm{x})$.

The potential operator can also be described from accurate quantum mechanical calculations. MRCI is among the quantum mechanical calculations able to describe the dissociation process as well as other spectroscopic constants in a very high level of accuracy. However, such methods are limited to be used in relatively small molecules. An interesting alternative to be applied in large system are the composite methods. Such alternative correspond to a combination of well-defined $\mathrm{ab}$ initio calculations to achieve an accurate total energy at a low computational cost when compared to high level calculations. These methods have been applied successfully in the calculation of thermochemical properties. $^{3-5}$ However, potential energy curves provided by such accurate methods have not been used to estimate spectroscopic properties.

The objective of this work is to explore potential curves of some diatomic molecules from a composite method to estimate spectroscopic constants. The composite method to be explored will be the Gaussian 3 theory or simply G3.6 In order to obtain the spectroscopic constants a new variational numerical procedure will be presented to solve the Schrödinger equation.

\section{Methods}

The general G3 energy is defined as:

$$
\begin{aligned}
& E \_G 3=E[M P 4 / 6-31 G(d)]+\Delta E(+)+\Delta E(2 d f, p)+ \\
& \Delta E(Q C I)+\Delta E(G 3 \text { large })+\Delta E(S O)+E(H L C)+E(Z P E)
\end{aligned}
$$

Where the correction are: $\Delta \mathrm{E}(+)$ for diffuse functions; $\Delta \mathrm{E}(2 \mathrm{df}, \mathrm{p})$ for polarization functions; $\Delta \mathrm{E}(\mathrm{QCI})$ for electronic correlation effects beyond fourth order perturbation theory using the method of quadratic configuration interaction; $\Delta \mathrm{E}(\mathrm{G} 3$ large) for larger basis set effects and for the nonadditivity caused by the assumption of basis set extensions for diffuse functions and higher polarization functions; spin- 
orbit correction, $\Delta \mathrm{E}(\mathrm{SO})$, for atomic species only; $\mathrm{E}(\mathrm{HLC})$, higher level correction, added to take into account remaining deficiencies in the energy calculations and finally E(ZPE) the zero-point energy at $0 \mathrm{~K}$ and thermal effects. In the present work, $\mathrm{E}(\mathrm{ZPE})$ is not being included to describe the potential curves. All the calculations were performed by using Gaussian09 software?.

From the G3 potential curves the time independent Schrödinger equation was solved using a form of variational quantum Monte Carlo (VQMC) method. The one-dimension mean value energy of a system is described as:

$$
E=\frac{\langle\psi|\ddot{H}| \psi\rangle}{\langle\psi \mid \psi\rangle}=\frac{\langle\psi(x)|\ddot{T}(x)+\ddot{V}(x)| \psi(x)\rangle}{\langle\psi(x) \mid \psi(x)\rangle}
$$

where the right side of Eq.(4) contains the kinetic (T) and potential operators $(\mathrm{V})$.

The kinetic energy operator is a second order derivative which can be described numerically by ${ }^{8}$

$$
\hat{T} \psi=\frac{1}{2 \mu} \frac{d^{2} \psi}{d x^{2}} \cong \frac{1}{2 \mu}\left[\frac{2}{h_{i}-h_{i-1}}\right]\left[\frac{\psi\left(x_{i+1}\right)-\psi\left(x_{i}\right)}{h_{i}}-\frac{\psi\left(x_{i}\right)-\psi\left(x_{i-1}\right)}{h_{i-1}}\right]
$$

where $i$ is the $i-t h m$ component of a discretized wavefunction vector, $\mu$ is the reduced mass of the system and $\mathrm{h}_{-} \mathrm{i}=\mathrm{x} \_(\mathrm{i}+1)$ $\mathrm{x} \_\mathrm{i}$. The potential energy operator will be described as set of discretized points obtained from the G3 calculation. After the application of the operators in an arbitrary and discretized wave function, Eq. (5) gives the energy of the system.

The systematic to get the result by VQMC is:?

i. Generates a random vector to be the initial wave function.

ii. Calculate the average energy using a discretized version of Eq.(4), Eq.(5) and the desired potential function.

iii. Select at random a single point of the vector and modify its value according to the equation:

$$
\psi\left(x_{-} i\right)=\psi\left(x \_i\right)+(1-r a n d) \delta
$$

where rand is a random number generator with uniform distribution between zero and 1 , and $\delta$ is a number which defines the range of change of the selected wave function.

iv. Recalculate the energy with the modified wave function using Eq. (4). If the new energy is lower than the first one, the modification is accepted. If it is not, the previous wave function is restored.

$\mathrm{v}$. The procedure is repeated until determined number of steps and/or the convergence factor is reached.

After the optimization of the ground state wave function, the first excited states can be obtained using the same procedure but preserving the orthogonality of the system. In this work the Gram-Schmidt method was used. ${ }^{10}$ The procedure is repeated to provide as many excited states as necessary.

After 106 steps of VQMC, an extra optimization of the meshes was carried out using the modified Simplex algorithm of Nelder and $\mathrm{Mead}^{11}$.

\section{Results and Discussion}

The harmonic oscillator, which has the potential energy operator shown in Eq. (1), was used as an initial test case. The boundaries of the domains have been defined between \pm 5 atomic units. The mass of the particle and the force constant have been set to 1 a.u. Table 1 shows ten states of the harmonic oscillators with 50,125 and 200 points.

Table 1. The tenth firsts states for the harmonic oscillator. Energies are in atomic units.

\begin{tabular}{|c|c|c|c|c|}
\hline State & 50pts & 125pts & 200pts & Exact \\
\hline E0 & 0.4987 & 0.4998 & 0.4999 & 0.5 \\
\hline E1 & 1.4934 & 1.4990 & 1.4996 & 1.5 \\
\hline E2 & 2.4823 & 2.4974 & 2.4990 & 2.5 \\
\hline E3 & 3.4672 & 3.4949 & 3.4982 & 3.5 \\
\hline E4 & 4.4463 & 4.4920 & 4.4981 & 4.5 \\
\hline E5 & 5.4196 & 5.4876 & 5.4972 & 5.5 \\
\hline E6 & 6.3896 & 6.4851 & 6.4981 & 6.5 \\
\hline E7 & 7.3582 & 7.4833 & 7.5021 & 7.5 \\
\hline E8 & 8.3347 & 8.4987 & 8.5240 & 8.5 \\
\hline E9 & 9.3420 & 9.5225 & 9.6037 & 9.5 \\
\hline
\end{tabular}

As expected, increasing the number of the points yield more accurate energies which tends to the exact value. The accuracy is reduced for higher excited states. The more orthogonal functions are required from the calculations, the more pronounced is the error propagation.

Figure 1 shows the first three harmonic wave functions. 


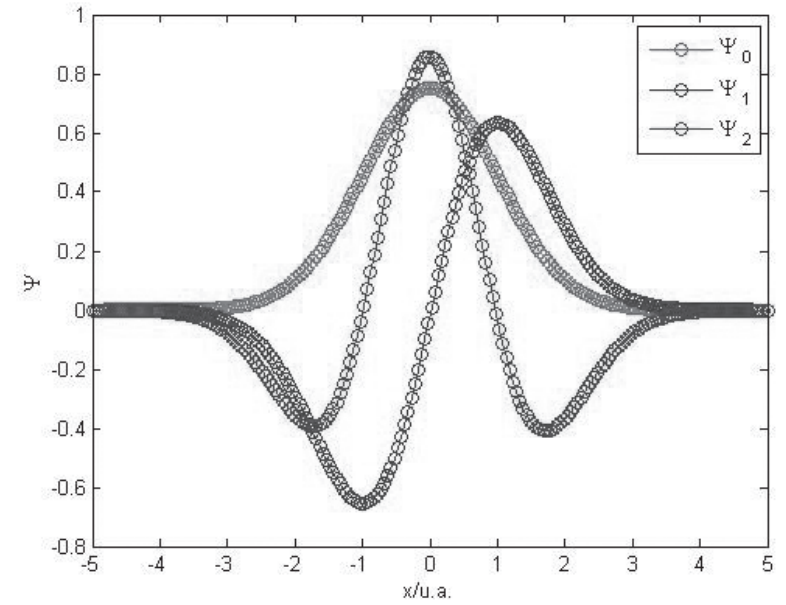

Figure 1. The three firsts wavefunctions of the harmonic oscillator.

The method generates not only accurate energies, but also well behaved wave functions as shown in Fig.1.

From the present uncertainty for harmonic oscillator, a mesh of 200 points will be used for the calculations involving the Morse and the G3 potentials. The reduced mass, dissociation energy and curvature for Morse's potential were taken from Herzberg, ${ }^{12}$ as well as the experimental energies. The same domain of the Morse's potential has been used to calculate the energies from the G3 theory. All meshes were constructed firstly with few points which were increased by spline interpolation. Table 3 shows the vibrational energies for $\mathrm{LiH}, \mathrm{H} 2$, HF, $\mathrm{HCl}, \mathrm{HBr}, \mathrm{O}_{2}$ and $\mathrm{Cl}_{2}$ by Morse's potential, $\mathrm{G} 3$ energies and experimental results. All the data are in atomic units.

Table 2 shows that the error in energy increases with the increases of the state of energy. By comparison, the error of the zero-th state of the $\mathrm{H}_{2}$ molecule, in Morse's potential, is $0.23 \%$, while the fourth state is $3.0 \%$. This enlargement of the error is explained by an error accumulation.

Some spectroscopic parameters were estimated by the equation:

$$
E(n)=\tilde{v}_{e}\left(n+\frac{1}{2}\right)-\tilde{x}_{e} \tilde{v}_{e}\left(n+\frac{1}{2}\right)^{2}
$$

where $\tilde{v} \tilde{}_{-}$is the fundamental frequency and $\tilde{x_{-}} \mathrm{e} \tilde{v_{-}} \mathrm{e}$ is the anharmonic constant. Table 3 shows the values of $v$ _e and $\tilde{x} \tilde{e}_{-} \tilde{v}_{-}$e from the calculations using the Morse and $\mathrm{G} 3$ potential, plus the experimental value.
Table 2. The first five vibrational energies calculated from the Morse and $\mathrm{G} 3$ potentials and experimental results. The simulation were carried out with discretized wave functions containing 200 points. Energies are in atomic units.

\begin{tabular}{|c|c|c|c|c|}
\hline & State & Morse & G3 & Exp.12 \\
\hline \multirow{5}{*}{$\mathrm{LiH}$} & E0 & 0.00317 & 0.00298 & 0.00317 \\
\hline & E1 & 0.00934 & 0.00880 & 0.00937 \\
\hline & E2 & 0.01528 & 0.01443 & 0.01535 \\
\hline & E3 & 0.02109 & 0.01985 & 0.02112 \\
\hline & E4 & 0.02720 & 0.02506 & 0.02668 \\
\hline \multirow{5}{*}{$\mathrm{H} 2$} & E0 & 0.00986 & 0.00990 & 0.00989 \\
\hline & E1 & 0.02871 & 0.02901 & 0.02884 \\
\hline & E2 & 0.04642 & 0.04688 & 0.04668 \\
\hline & E3 & 0.06297 & 0.06361 & 0.06341 \\
\hline & E4 & 0.08145 & 0.07921 & 0.07904 \\
\hline \multirow{5}{*}{$\mathrm{HF}$} & E0 & 0.00931 & 0.00936 & 0.00932 \\
\hline & E1 & 0.03000 & 0.02744 & 0.02736 \\
\hline & E2 & 0.04443 & 0.04468 & 0.04458 \\
\hline & E3 & 0.06072 & 0.06111 & 0.06098 \\
\hline & E4 & 0.07614 & 0.07672 & 0.07656 \\
\hline \multirow{5}{*}{$\mathrm{HCl}$} & E0 & 0.00674 & 0.00672 & 0.00675 \\
\hline & E1 & 0.01977 & 0.01972 & 0.01990 \\
\hline & E2 & 0.03222 & 0.03218 & 0.03256 \\
\hline & E3 & 0.04408 & 0.04409 & 0.04475 \\
\hline & E4 & 0.05554 & 0.05530 & 0.07365 \\
\hline \multirow{5}{*}{$\mathrm{HBr}$} & E0 & 0.00596 & 0.00599 & 0.00598 \\
\hline & E1 & 0.01749 & 0.01762 & 0.01764 \\
\hline & E2 & 0.02847 & 0.02880 & 0.02889 \\
\hline & E3 & 0.03893 & 0.03953 & 0.03972 \\
\hline & E4 & 0.04889 & 0.04978 & 0.05014 \\
\hline \multirow{5}{*}{$\mathrm{O} 2$} & E0 & 0.00357 & 0.00356 & 0.00359 \\
\hline & E1 & 0.01057 & 0.01061 & 0.01067 \\
\hline & E2 & 0.01738 & 0.01756 & 0.01765 \\
\hline & E3 & 0.02402 & 0.02438 & 0.02453 \\
\hline & $\mathrm{E} 4$ & 0.03046 & 0.03108 & 0.03129 \\
\hline \multirow{5}{*}{$\mathrm{Cl} 2$} & E0 & 0.00127 & 0.00122 & 0.00128 \\
\hline & E1 & 0.00375 & 0.00363 & 0.00382 \\
\hline & E2 & 0.00616 & 0.00600 & 0.00638 \\
\hline & E3 & 0.00852 & 0.00834 & 0.00892 \\
\hline & E4 & 0.01174 & 0.01063 & 0.01148 \\
\hline
\end{tabular}


Table 3. Numerical and experimental values of vibrational parameters, in $\mathrm{cm}-1$.

\begin{tabular}{rrrl|rrl}
\hline & \multicolumn{3}{c|}{$\tilde{v}_{\varepsilon}$} & \multicolumn{3}{|c}{$\tilde{x}_{e} \tilde{v}_{e}$} \\
\hline & Morse & G3 & Exp. ${ }^{12}$ & Morse & G3 & Exp. ${ }^{12}$ \\
$\mathrm{LiH}$ & 1342.9 & 1317.9 & 1405.6 & 19.12 & 42.92 & 23.20 \\
$\mathrm{H}_{2}$ & 4406.6 & 4431.9 & 4401.2 & 132.2 & 129.1 & 121.3 \\
$\mathrm{HF}$ & 4132.7 & 4157.5 & 4138.3 & 94.45 & 95.43 & 91.68 \\
$\mathrm{HCl}$ & 2972.5 & 2978.8 & 2990.9 & 59.17 & 62.64 & 52.82 \\
$\mathrm{HBR}$ & 2642.0 & 2650.4 & 2649.0 & 57.41 & 48.44 & 45.22 \\
$\mathrm{O}_{2}$ & 1577.8 & 1573.6 & 1580.2 & 20.40 & 12.44 & 11.98 \\
$\mathrm{Cl}_{2}$ & 535.15 & 538.24 & 559.72 & 0.183 & 4.422 & 2.675 \\
\hline
\end{tabular}

The calculated constants show that the errors do not follow a regular behavior. It implies that there are some situations that the Morse's potential fits better than G3, but this is not a rule.

\section{Conclusions}

The simulations performed in this work reveal that the present version of the variational principle associated with a Monte Carlo search is an accurate method to generate vibrational energies and well-behaved wave functions. The calculated spectroscopic parameters were usually considerably close to the experimental data with a small error. The approximate nature of both potentials tested may be pointed out as responsible for the deviations. Tests considering rigorous potential curves are under progress.

\section{Acknowledgments}

The authors are grateful for the financial support given from CAPES, CNPQ, FAPESP and FAEPEXUNICAMP.

\section{References}

1. F.L. Pilar. Elementary Quantum Chemistry. Mineola: Dover Publications SA, 2nd edition, 2001.

2. D.A. McQuarrie, J.D.Simon. Physical Chemistry: A Molecular Approach. New Delhi: Viva Books, 2013.

3. D. H. Pereira, A. F. Ramos, N. H. Morgon, and R. Custodio, J. Chem. Phys. 135, 034106 (2011).

4. D. H. Pereira, A. F. Ramos, N. H. Morgon, and R. Custodio, J.
Chem. Phys. 135, 219901 (2011).

5. C. M. R. Rocha, D. H. Pereira, N. H. Morgon, and R. Custodio, J. Chem. Phys. 139, 184108 (2013).

6. Larry A. Curtiss, Krishnan Raghavachari, Paul C. Redfern, Vitaly Rassolov, and John A. Pople. Journal Chemical Physics 109, 7764 (1998).

7. M. J. Frisch, G. W. Trucks, H. B. Schlegel, G. E. Scuseria, M. A. Robb, J. R. Cheeseman, G. Scalmani, V. Barone, B. Mennucci, G. A. Petersson, H. Nakatsuji, M. Caricato, X. Li, H. P. Hratchian, A. F. Izmaylov, J. Bloino, G. Zheng, J. L. Sonnenberg, M. Hada, M. Ehara, K. Toyota, R. Fukuda, J. Hasegawa, M. Ishida, T. Nakajima, Y. Honda, O. Kitao, H. Nakai, T. Vreven, J. A. Montgomery, Jr., J. E. Peralta, F. Ogliaro, M. Bearpark, J. J. Heyd, E. Brothers, K. N. Kudin, V. N. Staroverov, T. Keith, R. Kobayashi, J. Normand, K. Raghavachari, A. Rendell, J. C. Burant, S. S. Iyengar, J. Tomasi, M. Cossi, N. Rega, J. M. Millam, M. Klene, J. E. Knox, J. B. Cross, V. Bakken, C. Adamo, J. Jaramillo, R. Gomperts, R. E. Stratmann, O. Yazyev, A. J. Austin, R. Cammi, C. Pomelli, J. W. Ochterski, R. L. Martin, K. Morokuma, V. G. Zakrzewski, G. A. Voth, P. Salvador, J. J. Dannenberg, S. Dapprich, A. D. Daniels, O. Farkas, J. B. Foresman, J. V. Ortiz, J. Cioslowski, and D. J. Fox, Gaussian 09, Revision B.01, Gaussian, Inc., Wallingford CT, (2010).

8. B. Carnahan, H.A. Luther, J.O. Wikes. Applied Numerical Methods. New York: John Wiley \& Sons, 1969.

9. R. Custodio, M.R. Custodio, E.J. Creatto. Quim. Nova, 35, 2076, (2012).

10. R. Custodio. Quim. Nova, 9, 89, (1987).

11. J. A. Nelder and R. Mead, Comput. J. 7, 308 (1965).

12. K.P. Huber, G. Herzberg. Molecular Spectra and Molecular Structure IV. New York: Van Nostrand Reinhold Company, 1978.

\section{Maurício G. Rodrigues ${ }^{a}$ Régis C. Leal ${ }^{a, b}$ \& Rogério Custodio ${ }^{a}$}

a Universidade Estadual de Campinas (UNICAMP), 13083-970, Campinas - SP, Brazil.

'Instituto Federal de Educação, Ciência e Tecnologia do Rio Grande do Norte (IFRN), Campus Nova Cruz, 59215-000, Nova Cruz-RN, Brazil. 